\title{
Hypophyseal Portal Blood Collection for Analysis of GnRH Neurosecretion in Shiba Goats
}

\author{
Tomomi TANAKA, Natsuki OHGATA, Hideo KAMOMAE, \\ Yukari TAKEUCHI'), Yuji MORI') and Fred J KARSCH ${ }^{2)}$
}

\author{
Laboratory of Veterinary Reproduction, Tokyo University of Agriculture and \\ Technology, Saiwai-cho 3-5-8, Fuchu, Tokyo 183, \\ 1) Laboratory of Veterinary Ethology, The University of Tokyo, and \\ 2) Reproductive Science Program, The University of Michigan
}

\begin{abstract}
Sequential sampling of hypophyseal portal blood was performed in the Shiba goat. The technique for portal blood collection originally developed for sheep was adapted with some modification. Portal blood was withdrawn by lesioning a part of the pituitary portal vessels via a collection apparatus placed beforehand at the rostral surface of the anterior pituitary gland. In four ovariectomized goats, portal blood was collected successively at 5 min intervals for several hours to examine the neurosecretory dynamics of hypothalamic GnRH. A pulsatile pattern of GnRH discharge into the pituitary portal circulation was clearly observed. Mean \pm SD inter-pulse intervals in each of the 4 animals were $29.0 \pm 2.0 \mathrm{~min}, 38.8 \pm 2.2 \mathrm{~min}, 29.3 \pm 1.7 \mathrm{~min}$ and $23.9 \pm 2.1 \mathrm{~min}$. Each GnRH pulse was associated with an LH pulse, and temporal correlation between the GnRH and LH pulses was consistent despite differing pulse frequencies among individual animals. Thus the technique for continuous pituitary portal blood collection from conscious goat has become available.
\end{abstract}

Key words: Hypophyseal portal blood, GnRH, LH, Goat.

(J. Reprod. Dev. 43: 101-106, 1997)

I $\mathrm{t}$ has been established that a number of hypothalamic hormones such as gonadotropin-releasing hormone $(\mathrm{GnRH})$, corticotropin-releasing hormone and thyrotropin releasing hormone are released at the median eminence into the hypophyseal portal vessels to control the hormone secretion from the anterior pituitary gland, which then regulate the systemic endocrine system [1]. In most previous studies, the hypothalamic neuroendocrine function was assessed indirectly by examining the profiles of pituitary hormones in the peripheral circulation.

The hypophyseal portal blood collection technique has been developed for direct monitoring of

Accepted for publication: January 13, 1997

Correspondence: T. Tanaka the pattern of hypothalamic hormone release in ewes by Clarke and Cummins [2]. This technique enabled a prolonged sequential sampling of portal blood from conscious animals without disruption of anterior pituitary function. Several years later, Caraty and Locatelli $[3,4]$ modified the approach and could successfully collect portal blood samples more frequently and for longer period of time up to $48 \mathrm{~h}$ [5].

This study was performed to adapt the portal blood collection technique to the Shiba goat, which is widely used for research purposes and has proven to be an useful animal model to study neuroendocrine regulation of reproduction [6]. Since we have recently developed the method for electrophysiologically monitoring the GnRH pulse 
generator activity in this species [7], successful development of the portal blood collection procedure would provide the opportunity to combine these two techniques and then to assess the regulatory mechanism of the reproductive neuroendocrine system from novel points of view. The present paper reports the procedure for sequential collection of the pituitary portal blood and the pulsatile pattern of GnRH neurosecretion in the Shiba goat.

\section{Materials and Methods}

Long-term ovariectomized Shiba goats (20-30 kg body weight; $n=4$ ) were used. Implantation of a collection apparatus and the procedure for portal blood sampling were based upon the method originally developed in the sheep [5] with some modification for the Shiba goat.

\section{Surgery}

Following deprivation of feed and water for at least $24 \mathrm{~h}$, anesthesia was induced with intravenous administration of $2-3 \mathrm{ml}$ of a mixture solution of ketamine hydrochloride and xylazine hydrochloride (Veterinary Ketalar 50, Sankyo Inc., Japan; Celactal, Byerne Inc., Japan; 100:1 V/V), and maintained with halothane inhalation (1-2\% in oxygen, $500-700 \mathrm{ml} / \mathrm{min}$ ). The head of the animal was firmly attached to the stereotaxic apparatus [8], of which head holder and ear bars were modified for this purpose and held at nasal bones being approximately a $45^{\circ}$ angle relative to the surgical table. Horizontal skin incision of about $6 \mathrm{~cm}$ was made 2-3 mm dorsal to the supraorbital foramina, and then about $8 \mathrm{~cm}$ rostral incision was made downward from one end of the horizontal incision to a point midline on the frontal bone. After reflecting the skin to expose the frontal bone, a triangular piece of frontal bone at about $4 \mathrm{~cm}$ sides was removed with high speed drill. The exposed portion of dorsal nasal turbinates and nasal septum were removed using bone rongeurs and suction until the anterior face of the sphenoid bone appeared. Then a tunnel (about $10 \mathrm{~mm}$ diameter) was drilled through the spheroid bone until a portion of the optic chiasm appeared as a white horizontal band. The tunnel was then widened ventrally and advanced forward the anterior face of the pituitary with the aid of an operating microscope. The bone in front of the pituitary was carefully removed us- ing a drill and dental scalers, and the dura covering the pituitary was exposed. Then the square of dura (about $5 \times 5 \mathrm{~mm}$ ) was removed with the micro-knife. The collection apparatus $(90-100 \mathrm{~mm}$ length), which was made prior to surgery by joining 12 gauge needle (upper cannula) and 14 gauge needle (lower cannula) with dental acrylic and affixing a plastic cup (5-6 $\mathrm{mm}$ diameter) at one end, was placed into the tunnel in sphenoid bone and cemented in place with dental acrylic. The construction of this apparatus is described elsewhere [5]. After surgery, the apparatus was filled with heparinized saline $(100 \mathrm{IU} / \mathrm{ml})$ closed with plugs, and flushed with heparinized saline every day until sampling.

\section{Collection procedure}

After about 1 week recovery, the goat was tied loosely to a stanchion, where it could feed and rest during the experiment, and fitted with indwelling catheters in both jugular veins on the day prior to the sampling. On the day of sampling, the animal was systemically heparinized with an intravenous bolus of heparin (10,000 IU) administered 3 times at $30 \mathrm{~min}$ intervals. Then, after flushing the inside of collection apparatus with the saline, a sharp needle (16 gauge) was inserted into the upper cannula of the apparatus, and four or five small lesions (1$4 \mathrm{~mm}$ deep and distributed in a 120-180 circle) were made in the portal system at the level of the anterior face of the pituitary gland. Blood that flowed into the cup of the apparatus was withdrawn through the lower cannula using a peristaltic pump and collected as a series of small blocks separated by air, which was achieved by adjusting the rate of draw on the pump to be slightly greater than the rate of portal blood outflow. When the portal blood outflow was satisfactory $(>0.5 \mathrm{ml} / 5$ min), collection of peripheral blood was started through one jugular catheter and a solution of heparinized saline $(300 \mathrm{IU} / \mathrm{ml})$ was infused through the other jugular catheter at the same rate as peripheral blood collection (about $12 \mathrm{ml} / \mathrm{h}$ ). In the present study, matched portal and peripheral blood samples were obtained every $5 \mathrm{~min}$ for $3-4 \mathrm{~h}$. During the sampling, hematocrit of both portal and jugular samples was monitored hourly to check for possible contamination of portal blood with cerebrospinal fluid. Portal blood samples were collected in glass tubes containing $500 \mu \mathrm{l}$ bacitracin solution ( $3 \mathrm{mM}$, Bacitracin; Sigma Chemical, 
USA; in saline) by using a fraction collector and the samples were maintained in an ice bath. Portal blood volume was recorded for checking the collection rate and for evaluation of GnRH value after experiment. Portal blood samples were centrifuged and extracted within $1 \mathrm{~h}$ after collection (see below) as described previously [9]. Peripheral blood samples were centrifuged and frozen for LH assay. At the end of sampling, a jugular blood was taken into tubes containing bacitracin solution and processed as a portal sample for assay of $\mathrm{GnRH}$ to serve as the procedural blank. On the day after blood collection, the animals were killed by barbiturate overdose and the pituitary glands were removed and examined for the site of lesion.

\section{Hormone assays}

Plasma concentrations of GnRH were measured after methanol extraction using a previously described radioimmunoassay $[10,11]$ with small modification. A $750 \mu \mathrm{l}$ aliquot of portal sample mixed with methanol $(2 \mathrm{ml})$ and centrifuged (1580 G, $15 \mathrm{~min}$ ), and then supernatant was decanted and evaporated at $50 \mathrm{C}$ under a stream of air. Standards of GnRH were made up in a pool of goat peripheral plasma and were extracted in the same way. The dried extracts were re-dissolved in 250 $\mu \mathrm{l}$ assay buffer [PBS (pH 7.4, $0.1 \%$ gelatin, and $0.1 \%$ $\mathrm{NaNa}_{3}$ )]; $100 \mu \mathrm{l}$ of the extracts in duplicate were incubated with $100 \mu$ l antiserum (BDS antibody; $\times$ 200,000). After overnight incubation at 4 C, ${ }^{125}$ Ilabeled GnRH ( ${ }^{125}$ I-Luteinizing hormone releasing hormone; Amersham Life Science, UK) was added (approx. 10,000 cpm in $100 \mu \mathrm{l}$ ). After incubation for a further $6 \mathrm{~h}$, antibody-bound radioactivity was precipitated with ethanol $(2 \mathrm{ml})$. The supernatant after centrifugation at $1580 \mathrm{G}$ for $15 \mathrm{~min}$ was decanted and the precipitates counted in an automatic gamma counter. Recovery of known quantities of GnRH $(n=12)$ added to plasma averaged $82.4 \%$. Intra-assay coefficient of variation and the assay sensitivity were $6.4 \%$ and $0.29 \mathrm{pg} /$ tube, respectively.

LH was measured in triplicate aliquots of plasma by specific RIA described previously [12]. The sensitivity of the assay was $0.02 \mathrm{ng} /$ tube and intra- and inter-assay coefficients of variation were $5.9 \%$ and $10.3 \%$, respectively.

\section{Data analysis}

GnRH values were evaluated as mass of hor- mone collected per unit time ( $\mathrm{pg} / \mathrm{min}$ ) rather than concentration to minimize potential sources of error in portal blood collection technique such as contamination with non-portal blood or change in flow rate due to head position [13]. Pulses of GnRH and LH were analyzed with the pulsar algorithm [14].

\section{Results}

Blood samples were collected successfully from all four animals used in the experiment and there was no cerebrospinal fluid contamination of portal samples as judged by comparison of hematocrits in portal and jugular blood samples. The secretory pattern of $\mathrm{GnRH}$ in portal circulation and of $\mathrm{LH}$ in peripheral circulation in each of the 4 ovariectomized goats are shown in Fig. 1. In all animals, the pulsatile nature of GnRH secretion was clearly demonstrated. Further, each pulse of GnRH was associated with the initiation of a pulse of LH. The mean \pm SD inter-pulse intervals of $\mathrm{GnRH}$ in each of 4 animals were $29.0 \pm 2.0 \mathrm{~min}(\# 3), 38.8 \pm 2.2$ $\min (\# 5), 29.3 \pm 1.7 \mathrm{~min}(\# 7)$ and $23.9 \pm 2.1 \mathrm{~min}$ (\#8). The secretory pattern of GnRH was characterized by an abrupt increase, usually reaching the peak within one 5-min sampling period, followed by a rapid decrease to the baseline within 5-10 min.

The lesioning sites were found to be located in the anterior face of the pituitary. The concentration of GnRH in peripheral circulation never exceeded the level of assay sensitivity.

\section{Discussion}

There is a general agreement that the hypothalamic decapeptide hormone $\mathrm{GnRH}$ is released at the median eminence into the portal circulation in the pulsatile manner, which drives the pulsatile LH secretion from the anterior pituitary. However, the pulsatile pattern of GnRH neurosecretion can not be detected in peripheral blood because of a rapid turnover and dilution of GnRH in the peripheral circulation. The temporal relationship between GnRH pulses and LH pulses has been documented so far in the monkey $[15,16]$, sheep $[2-4,17]$, rabbit [18], rat $[19,20]$ and horse [21]. In the present study, we adapted the portal blood 

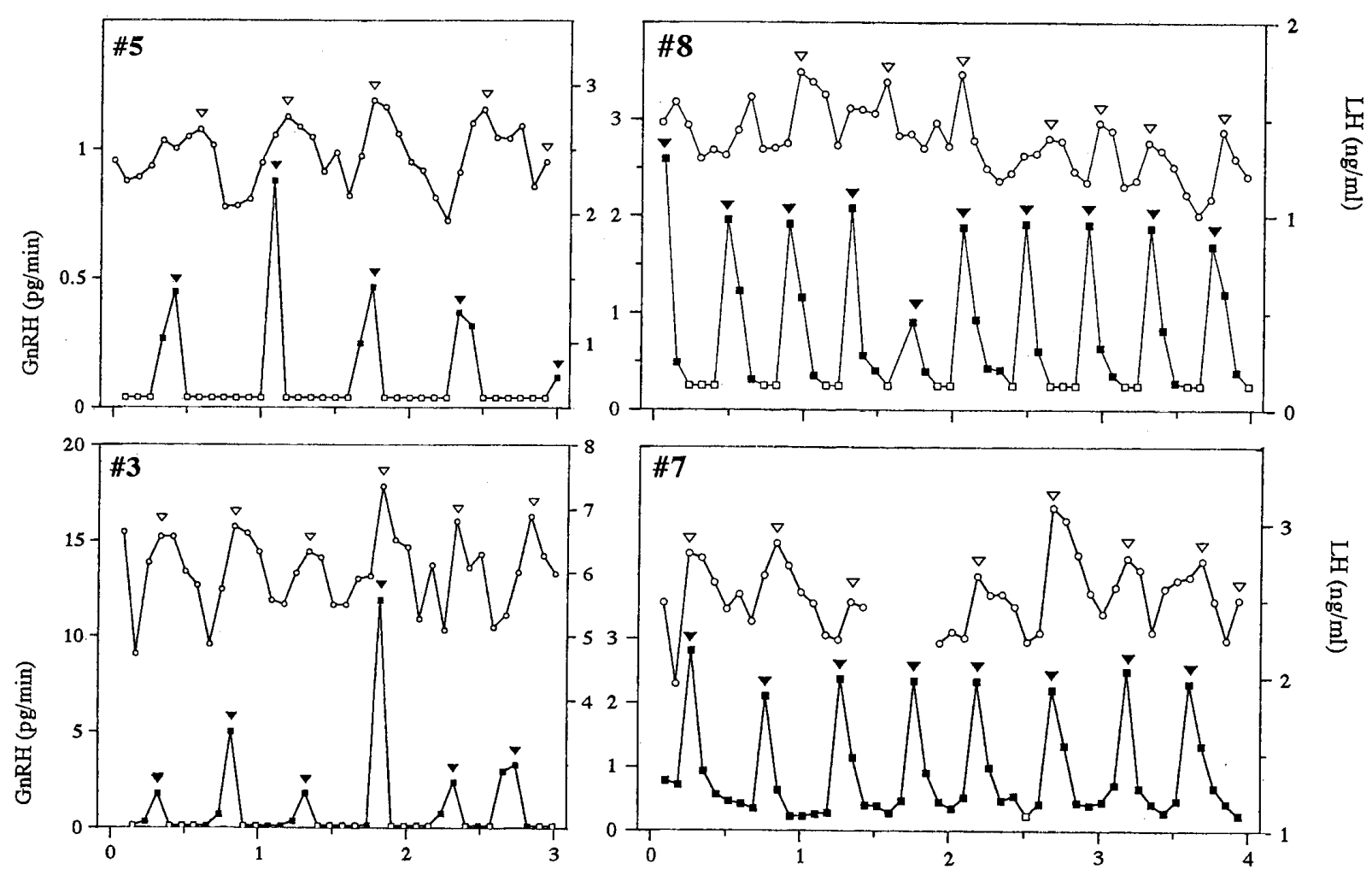

Time (hr)

Fig. 1. Patterns of GnRH in pituitary portal blood (squares in lower portion of each box) and of LH in the peripheral blood (circles in upper portion of each box) obtained at 5 min intervals for $3(\# 5, \# 3)$ or 4 (\#8, \#7) h from four ovariectomized Shiba goats. Open squares depict undetectable GnRH values. Solid and open triangles indicate the peak of pulses identified by the pulsar computer program for GnRH and LH, respectively.

sampling technique, which had been originally developed for sheep, for use in goats and confirmed that GnRH pulses were associated with $\mathrm{LH}$ pulses in all the ovariectomized goats examined. The results were very much the same as those previously reported in sheep [2-4].

Moenter et al. [22] examined the moment-to-moment change in GnRH in pituitary portal blood during each pulse in pituitary portal blood sampled at $30 \mathrm{sec}$ interval from ovariectomized ewes. They demonstrated that the shape of most GnRH pulses approximated a square wave with an abrupt ascent, a release period averaging about $5 \mathrm{~min}$, and a precipitous descent to the pre-pulse baseline value. In the present study, GnRH pulses in ovariectomized goats were found to be characterized by abrupt increases and decreases, with very low levels during the inter-pulse period, which is consistent with the view that GnRH is released into the portal circulation only for a few min at the time of pulses in ovariectomized ewes. Moreover, these findings are consistent with the results obtained from our previous electrophysiological study. Specifically, we observed that the characteristic increases of the hypothalamic multiple unit activity specifically associated with pulsatile $\mathrm{LH}$ secretion (MUA volleys) were abrupt and brief, lasting about $3 \mathrm{~min}$ in the ovariectomized goat [7]. These results further support the view that the MUA volley is the electrophysiological manifestation of the hypothalamic GnRH pulse generator activity which controls the pattern of actual GnRH neurosecretion. The present results were obtained from ovariectomized animals. Further investigation is needed to clarify how the pulsatile pattern of GnRH neurosecretion changes under different physiological circumstances and in response to various hormonal manipulations. 
In the present study, we successfully adapted the portal blood collection technique for the goat and demonstrated that the pattern of GnRH was pulsatile in pituitary portal circulation. This technique will also be feasible for monitoring the moment-to-moment dynamics of various hypothalamic hormone besides GnRH. In addition, by combining the procedures for portal blood collection and electrophysiological monitoring of $\mathrm{GnRH}$ pulse generator activity, a novel approach would become available to investigate the neuroendocrine control mechanism of the reproductive endocrine system in the ruminant species.

\section{Acknowledgments}

We are grateful to Drs. Kei-Ichiro Maeda and Douglas L Foster for affording the opportunity to undertake this study with the travel grant from Japan Society for the Promotion of Science (JSPS). We are thankful to Dr. Alain Caraty for providing the GnRH antibody. We thank Takehisa Yamamoto for his assistance during the sampling. This work was supported in part by grants from Ministry of Education, Science, Sports and Culture, Japan.

\section{References}

1. Everitt BJ, Hokfelt T. Neuroendocrine anatomy of the hypothalamus. In: Lightman SL, Everitt BJ (eds.), Neuroendocrinology. London: Blackwell Scientific Publications; 1986: 5-31.

2. Clarke IJ, Cummins JT. The temporal relationship between gonadotropin releasing hormone (GnRH) and luteinizing hormone (LH) secretion in ovariectomized ewes. Endocrinology 1982; 111: 1737-1739.

3. Caraty A, Locatelli A. Effect of time after castration on secretion of LHRH and LH in the ram. J Reprod Fertil 1988; 82: 263-269.

4. Caraty A, Locatelli A, Martin GB. Biphasic response in the secretion of gonadotropin-releasing hormone in ovariectomized ewes injected with oestradiol. J Endocrinol 1989; 123: 375-382.

5. Caraty A, Locatelli A, Moenter SM, Karsch FJ. Sampling of hypophyseal portal blood of conscious sheep for direct monitoring of hypothalamic neurosecretory substances. Method in Neuroscience 1994; 20: 162-183.

6. Mori Y. Central integration of photoperiodicity for gonadotropin release in ruminants. In: Yokoyama A (ed.), Brain Control of the Reproductive System. Tokyo: Japan Sci. Soc. Press / CRC Press; 1992: 93118.

7. Mori $Y$, Nishihara M, Tanaka T, Shimizu T, Yamaguchi M, Takeuchi Y, Hoshino K. Chronic recording of electrophysiological manifestation of the hypothalamic gonadotropin-releasing hormone pulse generator activity in the goat. Neuroendocrinology 1991; 53: 392-395.

8. Mori Y, Takeuchi Y, Shimada M, Hayashi S, Hoshino K. Stereotaxic approach to hypothalamic nuclei of the Shiba goat with radiographic monitoring. Jpn J Vet Med Sci 1990; 52: 339-349.

9. Jeffcoate SL, Greenwood RH, Holland DT. Blood and urine clearance of luteinizing hor- mone releasing hormone in man measured by radioimmunoassay. J Endocrinol 1974; 60: 305-314.

10. Caraty A, Locatelli A, Schanbacher B. Augmentation, by naloxone, of the frequency and amplitude of LH-RH pulses in hypothalamo-hypophyseal portal blood in the castrated ram. $C R$ Acad $S c i$ 1987; 305: 369-374 (In French).

11. Moenter SM, Caraty A, Karsch FJ. The estradiolinduced surge of gonadotropin-releasing hormone in the ewe. Endocrinology 1990; 127: 1375-1384.

12. Mori Y, Kano Y. Changes in plasma concentrations of $\mathrm{LH}$, progesterone and oestradiol in relation to the occurrence of luteolysis, oestrus and time of ovulation in the Shiba goat (Capra hircus). J Reprod Fertil 1984; 72: 223-230.

13. Karsch FJ, Cummins JT, Thomas GB, Clarke IJ. Steroid feedback inhibition of pulsatile secretion of gonadotropin-releasing hormone in the ewe. Biol Reprod 1987; 36: 1207-1218.

14. Merriam GR, Wachter KW. Algorithms for the study of episodic hormone secretion. Am J Physiol 1982; 243: E310-E318.

15. Levine JE, Norman RL, Gliessman PM, Oyama TT, Bangsberg DR, Spies HG. In vivo gonadotropin-releasing hormone release and serum luteinizing hormone measurements in ovariectomized, estrogen-treated rhesus macaques. Endocrinology 1985; 117: 711-721.

16. Van Vugt DA, Diefenbach WD, Alston E, Ferin M. Gonadotropin-releasing hormone pulses in third ventricular cerebrospinal fluid of ovariectomized rhesus monkeys: Correlation with luteinizing hormone pulses. Endocrinology 1985; 117: 1550-1558.

17. Levine JE, Pau K-YF, Ramirez VD, Jackson GL. Simultaneous measurement of luteinizing hormone and luteinizing hormone release in 
unanesthetized, ovariectomized sheep. Endocrinology 1982; 111: 1449-1455.

18. Pau K-YF, Orstead KM, Hess DL, Spies HG. Feedback effects of ovarian steroids on the hypothalamic-hypophyseal axis in the rabbit. Biol Reprod 1986; 35: 1009-1023.

19. Levine JE, Bayer-Dantoin AC, Besecke LM, Conagham LA, Leagn SJ, Meredith JM, Strobl FJ, Urban JH, Vogeisong KM, Wolfe AM. Neuroendocrine regulation of the luteinizing hormone-releasing hormone pulse generator in the rat. Recent Prog Horm Res 1991; 47: 97-153.

20. Levine JE, Duffy MT. Simultaneous measurement of luteinizing hormone (LH)-releasing hormone,
$\mathrm{LH}$, and follicle-stimulating hormone release in intact and short-term castrate rats. Endocrinology 1988; 122: 2211-2221.

21. Irvine CHG, Alexander SL. Secretory patterns and rates of gonadotropin-releasing hormone, folliclestimulating hormone, and luteinizing hormone revealed by intensive sampling of pituitary venous blood in the luteal phase mare. Endocrinology 1993; 132: 212-218.

22. Moenter SM, Brand RM, Midgley AR, Karsch FJ. Dynamics of Gonadotropin-releasing hormone release during a pulse. Endocrinology 1992; 130: 503-510. 